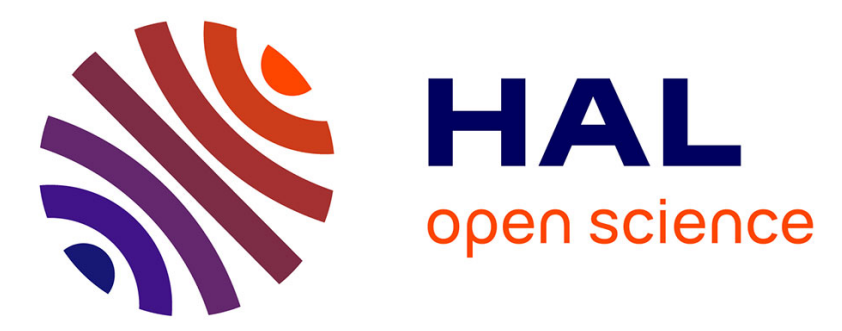

\title{
Stacking denoising auto-encoders in a deep network to segment the brainstem on MRI in brain cancer patients: a clinical study
}

Jose Dolz, Nacim Betrouni, Mathilde Quidet, Dris Kharroubi, Henri A. Leroy, Nicolas Reyns, Laurent Massoptier, Maximilien Vermandel

\section{To cite this version:}

Jose Dolz, Nacim Betrouni, Mathilde Quidet, Dris Kharroubi, Henri A. Leroy, et al.. Stacking denoising auto-encoders in a deep network to segment the brainstem on MRI in brain cancer patients: a clinical study. Computerized Medical Imaging and Graphics, 2016, 10.1016/j.compmedimag.2016.03.003 . hal-01318076

\section{HAL Id: hal-01318076 \\ https://hal.science/hal-01318076}

Submitted on 19 May 2016

HAL is a multi-disciplinary open access archive for the deposit and dissemination of scientific research documents, whether they are published or not. The documents may come from teaching and research institutions in France or abroad, or from public or private research centers.
L'archive ouverte pluridisciplinaire HAL, est destinée au dépôt et à la diffusion de documents scientifiques de niveau recherche, publiés ou non, émanant des établissements d'enseignement et de recherche français ou étrangers, des laboratoires publics ou privés. 


\title{
Stacking denoising auto-encoders in a deep network to segment the brainstem on MRI in brain cancer patients: a clinical study
}

\author{
Jose Dolz ${ }^{\mathrm{a}, \mathrm{b}, *}$, Nacim Betrouni ${ }^{\mathrm{b}}$, Mathilde Quidet $^{\mathrm{b}}$, Dris Kharroubi ${ }^{\mathrm{b}}$, Henri A. \\ Leroy $^{\mathrm{b}, \mathrm{c}}$, Nicolas Reyns ${ }^{\mathrm{b}, \mathrm{c}}$, Laurent Massoptier ${ }^{\mathrm{a}}$, Maximilien Vermandel ${ }^{\mathrm{b}, \mathrm{c}}$ \\ ${ }^{a}$ AQUILAB, Loos-les-Lille, France \\ ${ }^{b}$ Univ. Lille, Inserm, CHU Lille, U1189 - ONCO-THAI - Image Assisted Laser Therapy \\ for Oncology, F-59000 Lille, France \\ ${ }^{c}$ Neurosurgery Department, University Hospital Lille, Lille, France
}

\begin{abstract}
Delineation of organs at risk (OARs) is a crucial step in surgical and treatment planning in brain cancer, where precise OARs volume delineation is required. However, this task is still often manually performed, which is time-consuming and prone to observer variability. To tackle these issues a deep learning approach based on stacking denoising auto-encoders has been proposed to segment the brainstem on magnetic resonance images in brain cancer context. Additionally to classical features used in machine learning to segment brain structures, two new features are suggested. Four experts participated in this study by segmenting the brainstem on 9 patients who underwent radiosurgery. Analysis of variance on shape and volume similarity metrics indicated that there were significant differences $(\mathrm{p}<0.05)$ between the groups of manual annotations and automatic segmentations. Experimental evaluation also showed an overlapping higher than $90 \%$ with respect to the ground truth. These results are comparable, and often higher, to those of the state of the art segmentation methods but with a considerably reduction of the segmentation time.
\end{abstract}

Keywords: Deep learning, MRI segmentation, brain cancer, machine learning.

\section{Introduction}

Cancer is a leading cause of death and disability worldwide, accounting for 14.1 million of new cancer cases and 8.2 million deaths in 2012 [1]. Among available techniques to treat brain tumors, radiotherapy and radio surgery have

5 often become the selected treatment, especially when others techniques such as surgery or chemotherapy might not be applicable. To constrain the risk of severe toxicity of critical brain structures, i.e. the organs at risk (OARs),

${ }^{*}$ Corresponding author: jose.dolz.upv@gmail.com 
the volume measurements and the localization of these structures are required. Among available image modalities, magnetic resonance imaging (MRI) images are extensively used to segment most of the OARs, which is performed mostly manually nowadays.

However, manual delineation of large brain structures, such as the brainstem, could be prohibitively time-consuming, and could never be reproducible during clinical routines [2, 3], leading to substantial inconsistency in the segmentation.

15 Particularly, in the case of the brainstem, variability on delineation is especially notorious in the area where the brainstem meets with the cerebellum in the lower pons, and where no significant contrast boundary is present. Thus, image segmentation has become a central part in the radiation treatment planning (RTP), being often a limiting step of it. Therefore, automatic or semi-automatic 20 segmentation algorithms are highly recommended in order to surmount such disadvantages.

Segmentation of brain structures have been mainly approached by using atlas-based methods [4, 5]. Although good performance has been reported, evaluation of these methods has been made on control and on several mental 25 disorders patients, such as Schizophrenia or Alzheimer. However, in brain cancer context, the presence of tumors may deform other structures and appear together with edema that changes intensity properties of the nearby region, making the segmentation more challenging. To evaluate the performance of atlas-based approaches to segment the brainstem, among other structures, in

30 such situations, some work have been recently presented in the context of brain cancer treatment [2, 3, 6. In [2, a large study that engaged 8 experts and included 20 patients reported mean Dice similarity coefficient (DSC) respect to the ground truth generated of nearly 0.85. Euclidean average maximum distances ranged from - to $+5.4 \mathrm{~mm}$ (inside and outside). Similar to this work, in 3, an 35 atlas-based segmentation was evaluated on 6 patients and compared to 7 expert delineations. Comparison between experts and automatic results showed that brainstem segmentation volume generated by the automatic approach lay in 5 out of 6 cases between the variations of the experts, with a volume underestimation ranging from $-14 \%$ to $-2 \%$ in these patients. In the work of Isambert et

40 al. 6], brainstem automatic contours were accepted for clinical routine with a volume underestimation of $-15 \%$ with respect to the manual segmentation and mean DSC of 0.85 ranging from 0.8 to 0.88 . Although the brainstem has been often successfully segmented, with DSC values typically greater than 0.8 , segmentation time reported has been always above several minutes. In addition 4 to time constraints derived from the registration step, atlas-based methods require large variation on the atlases to capture anatomical variability in target patients.

To overcome difficulties of atlas-based approaches, we considered the use of denoising auto-encoders in the presented work. Deep learning has already been 50 used to segment some tissue or organs in the medical domain others than the brainstem [7, 8]. In these approaches, two or three-dimensional image patches are commonly fed into the deep network, which unsupervisedly learns the best features representation of the given patches. Computed neurons' weights are 
then re-fined during a second supervised step. However, valuable information inherited from classical machine learning approaches to segment brain structures is not included in these input vectors. This knowledge may come in the form of likelihood voxel values or voxel location, for example, which is greatly useful to segment structures that share similar intensity properties.

In the present work, we propose a deep neural network formed by stacking de${ }_{60}$ noising auto-encoders (SDAE) as alternative to atlas-based methods to segment the brainstem. Additionally, we compare the results with a well-known machine learning classifier, support vector machines (SVM). Furthermore, instead of using patches from single or multi-modality MR images, we use hand-crafted features as input of the network. Lastly, as extension to features typically employed in machine learning approaches to segment brain structures 9, 10, 11, we propose the inclusion of two new features in the features vector of the classifier when segmenting the brainstem. Our main contribution is, therefore, a new and practical application of SDAE, which recently produced outstanding results in solving some medical image problems, such as classification or segmentation.

\section{Methods and materials}

\subsection{Features used in the classification}

The most influencing factor in realizing a classifier with high generalization ability is the set of features used. A poor selection of the features to be used in the classifier may lead to unsatisfactory results. Intensity based approaches have been largely employed to segment objects of interest in the medical field. Nevertheless, image intensity information solely is not good enough for distinguishing different subcortical structures since most of them share similar intensity patterns in MRI. To address such problem, in learning based segmentation methods, more discriminative features are often extracted from MRI 9, 10, 11.

80 In addition to image intensity values (IIV) of the neighboring voxels, spatial and probabilistic information is often used in the creation of a classifier. Texture information, i.e. IIV related information, can be captured in many ways. For instance, Powell et al. 9] captured texture information by using 8 IIV along the largest gradient, including the voxel under examination. Additionally, a probabilistic map, IIVs along each of the three orthogonal axes and the probability of being part of a particular structure were used as features for each sample. In [10], a slightly modified input vector was adapted to increase the performance of a learning-based scheme. In their work, a modified spatial location of the spherical coordinates of the voxel $v$ and a neighborhood connection

90 based on gradient descent were used. While the former aided to reflect symmetry of brain, the latter was used for directional consistency. More recently, in [11, different IIVs configurations were compared to segment the brainstem, in addition to probability values and spherical coordinates of the voxel under examination. Configurations proposed in this work included cubic patches of

95 different sizes, orthogonal crosses and, as in 9, voxels along the direction of maximum gradient. 


\subsubsection{Geodesic transform map.}

To encourage spatial regularization and contrast-sensitivity, geodesic distance transform map (GDTM) of the input image is used as additional feature. The addition of GDTM in the features vector used by the classifier exploits the ability of seed-expansion to fill contiguous, coherent regions without regard to boundary length. As explained in the work of Criminisi et al. [12, given an image $I$ defined on a 2D domain $\psi$, a binary mask $M$ (with $M(\mathrm{x}) \in\{0,1\} \forall \mathrm{x}$ ) and an "object" region $\Omega$ with $\mathrm{x} \in \Omega \Longleftrightarrow M(\mathrm{x})=0$, the unsigned geodesic

distance of each pixel $\mathrm{x}$ from $\Omega$ is defined as:

$$
\begin{aligned}
& D(x ; M, \nabla I)=\min _{\left\{x^{\prime} \mid M\left(x^{\prime}\right)=0\right\}} d\left(x, x^{\prime}\right), \quad \text { with } \\
& d(a, b)=\min _{\Gamma \in \mathcal{P}_{a, b}} \int_{0}^{1} \sqrt{\left\|\Gamma^{\prime}(s)\right\|^{2}+\gamma^{2}(\nabla I \cdot u)^{2} d s}
\end{aligned}
$$

with $\mathcal{P}_{a, b}$ the set of all paths between the points a and $\mathbf{b}$, and $\Gamma(\mathrm{s}): \Re \rightarrow$ $\Re^{2}$ indicating one such path, which is parametrized by $s \in[0,1]$. The term $u$ represents the unit tangent vector, which is defined as $u=\Gamma^{\prime}(s) / \mid \Gamma(s) \|$. Fig. 1 shows an example of how to compute the GTDM of an image given a binary mask.
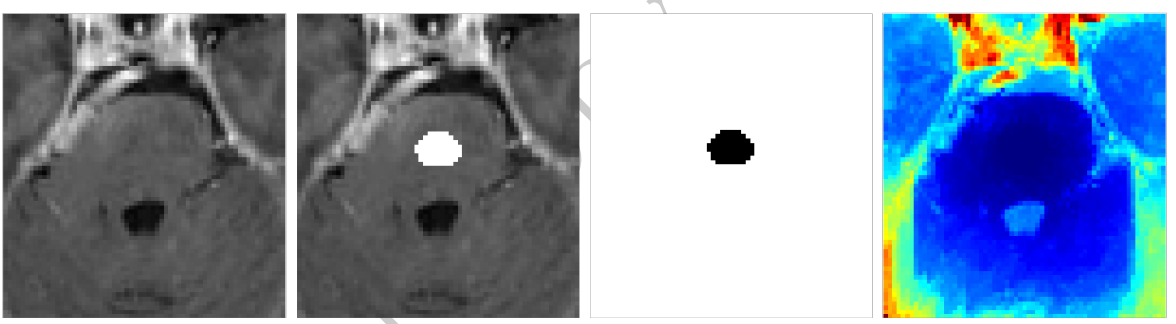

Figure 1: Geodesic distance transform map: a) axial MR view of the brainstem, b) mask obtained from the probability brainstem map (in white) over the MRI axial slice, c) binary mask used to obtain the GDTM, and d) computed GDTM.

\subsubsection{Local binary pattern.}

In order to catch neighborhood appearance of the voxel under examination with the fewest number of features, Local Binary Patterns (LBP) are investigated. The idea of LBP is to give a pattern code to each voxel. Particularly, an extended version of 3D-LBP presented by [13 is proposed. In their work, classical LBP were adapted by selecting the 6 nearest voxels and ordering them to create the encoding patterns. By encoding patterns in that manner, $2^{6}=64$ possible patterns would be created. However, those 64 possible combinations were merged in 10 different groups according to geometrical similarities (Figure 2). In accordance with this classification, each group is filled with patterns that have the same number of neighbor voxels with a gray level higher than the 
central voxel $c$. Thus, rotation invariance in each group is kept. These groups are defined with (Figure $2, \mathrm{~b})$ :

$$
\operatorname{card}(c)=\sum_{i=0}^{P-1} s\left(g_{i}-g_{c}\right), \quad \text { with } \quad s(x)= \begin{cases}1 & \text { if } x \geq 0 \\ 0 & \text { else }\end{cases}
$$

where $\mathrm{P}=6$ is the number of neighboring voxels and $R=1$ or $R=2$ the distance between central voxel $c$ and its neighbors $i$. By using $\mathrm{R}=1,2$ micro and macrostructure appearance of the texture are captured in the 3D-LBP. In equation $3, \operatorname{card}(c)$ gives the number of neighbors with a higher gray level than the central voxel $c$.

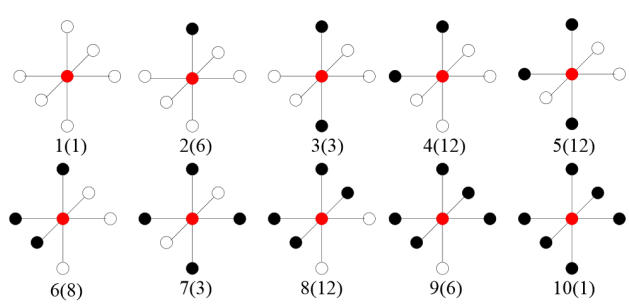

(a)

\begin{tabular}{c|c|l}
\hline$L B P_{P, R}^{3 d}$ & card $(c)$ & condition \\
\hline 1 & 0 & \\
2 & 1 & \\
3 & 2 & opposite voxels \\
4 & 2 & bend voxels \\
5 & 3 & voxels on the same plane \\
6 & 3 & voxels on different planes \\
7 & 4 & voxels on the same plane \\
8 & 4 & voxels on different planes \\
9 & 5 & \\
10 & 6 & \\
\hline
\end{tabular}

(b)

Figure 2: (a). Merging the 64 possible patterns into 10 groups. Number of different patterns for each group is indicated in brackets.(b).Definition of the 10 groups of patterns 13 .

In addition to the encoded value for the 3D patch structure proposed by [13], an additional texture value is included. Let $g_{h i g h}$ the gray values that are higher than the gray value of the center voxel $c$ in the 3D-LPB (Figure 2). Similarly, let's denote $g_{l o w}$ to the gray values that are lower than the gray value of the center voxel $c$ in the 3D-LPB. Then, the texture value added to the encoded structure value is defined as:

$$
\text { Texture }_{\text {val }}=\text { mean } \sum_{i=0}^{m} g_{\text {high }}(i)-\text { mean } \sum_{i=0}^{n} g_{\text {low }}(i)
$$

where $m$ and $n$ are the number of neighboring voxels with higher and lower LBP in the features vector will lead to 4 new features: 3D-LBP and Texture val for $\mathrm{R}=1$ and 2 .

\subsubsection{Composition of the input features vector.}

Different combinations of intensity values of the neighborhood of a given that work, the intensity value of the voxel under investigation and the intensity values of the 8 voxel along the direction of maximum gradient reported the best trade-off in terms of segmentation similarity and computational cost. Therefore, 
this configuration is used in the present work to capture intensity information from the MRI image. Additionally, to better understand the pattern of each voxel with respect to its neighborhood, while feeding the minimum amount of information into the features vector, the 3D-LPB introduced in section 2.1.2 is used. Its use leads to 2 values, one for the pattern and one for the texture, at each of the two radius used $(\mathrm{R}=1,2)$. To complete the features vector, the value 145 at the voxel location of the probability map, geodesic distance transform map and image gradient are used, as well as the spherical coordinates at its location (Figure 3). Thus, a vector containing a total of 19 feature values is used to characterize each sample.

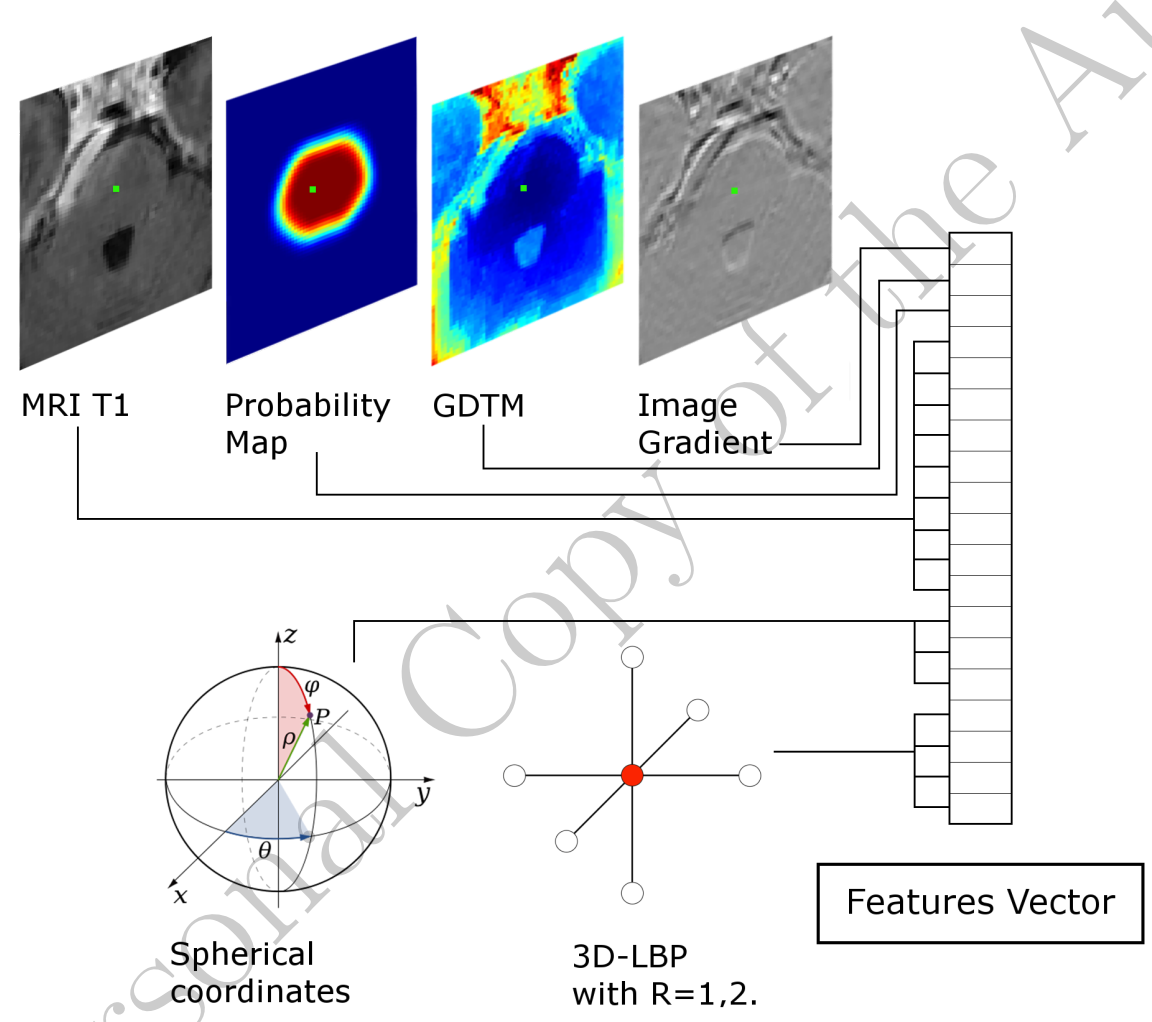

Figure 3: Features fed into the features vector for each sample (voxel). In green is pointed out the voxel under examination.

In order to provide a robust classifier, features that compose the input array must show discriminative power. This means that each of these features must be complementary to the others, providing additional information that helps in the classification task. To visually demonstrate that features incorporated into the features array are uncorrelated, correlations between some pairs of features are plotted (Fig. 4). Image intensity is combined with three of the proposed features -probability map, geodesic transform map and image gradient-. 
Thus, we investigate whether they bring valuable information to the knowledge provided by intensity values to perform the classification. Analysis conducted on each pair of features showed that including them, in addition to intensity information, helps for distinguishing between classes.

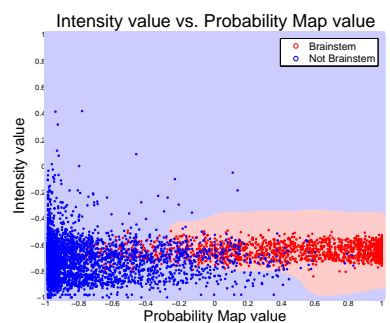

a

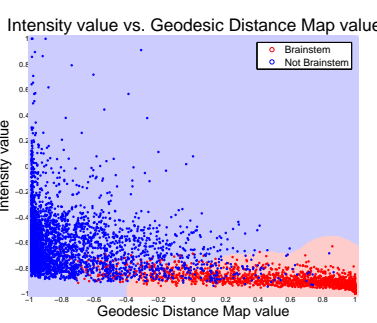

b

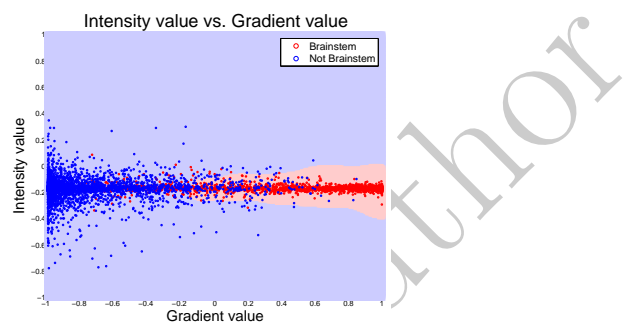

C

Figure 4: Decision boundary plots for two classes by using solely intensity information and : a) probability map values, b) geodesic distances map values and c) gradient values.

\subsection{Support vector machines}

Support vector machines (SVM), often called kernel-based methods, have been extensively studied and applied to several pattern classification and function approximation problems. Basically, the main idea behind SVM is to find the largest margin hyperplane that separates two classes. The minimal distance gin. Thus, the optimal hyperplan is the which represents the largest separation between the classes. This will be the line such that the distances from the closest point in each of the two groups will be farthest away. The training samples that lie on the margin are referred as Therefore, support vectors define the location of the separating hyperplane, being located at the boundary of their respective classes. By employing kernel transformations to map the objects from their original space into a higher dimensional feature space [14, SVM can separate objects which are not linearly 175 separable (Figure 5). Their good generalization ability and their capability to successfully elassify non-linearly separable data have led to a growing interest on them for classification problems.

\subsection{Deep Learning based classification scheme}

Classification problem is solved in this work by using deep learning. This 180 technique learns hierarchical correlations between feature representations in a given dataset through a semi-supervised learning approach [15]. Hence, the proposed approach follows a hybrid architecture which unsupervisedly learns the features' representation of the hand-crafted features followed by a supervised fine tuning of the parameters of the deep network. 
Classification by mapping features into higher dimensions may become easier

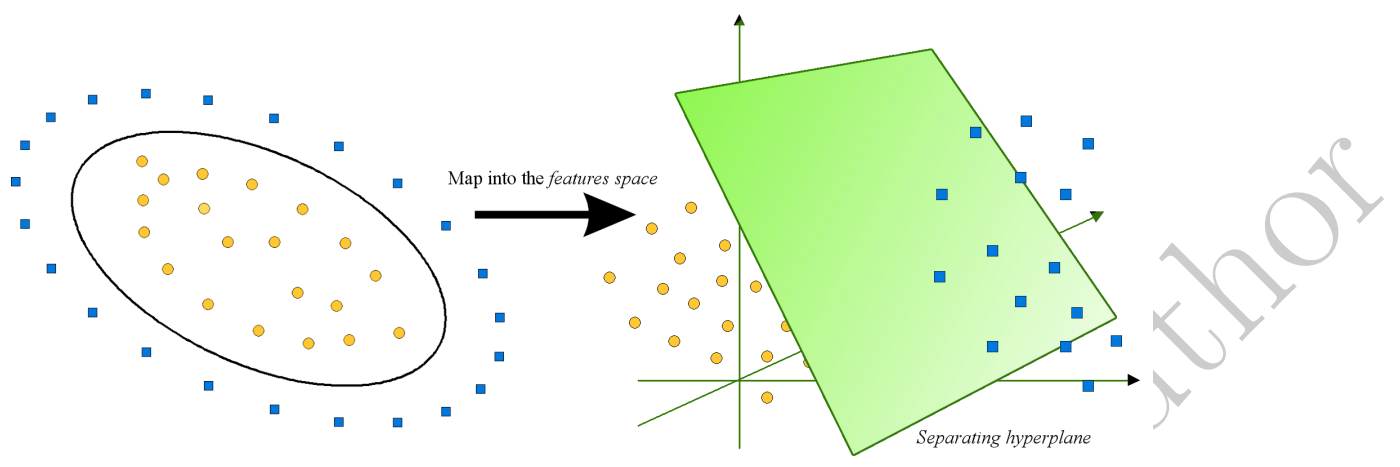

Complex classification in low dimensions

Classification becomes simpler in higher dimensions

Figure 5: SVM and hyperplane. Mapping features into a higher dimensionality may make the classification simpler.

The deep network used in the proposed classification scheme is formed by stacking DAEs (Fig. 6). Weights between layers of the network are initially learned via the unsupervised pre-training step (Sec. 2.3.1). Once all the weights of the network are unsupervisedly computed, a supervised refinement is carried out by using the labeled classes, and final values of the network' weights are updated (Sec. 2.3.2).

\subsubsection{Unsupervised pre-training of DAEs}

Classical auto-encoders (AE) have been recently developed in the deep learning literature in different forms [16]. In its simplest representation, an $\mathrm{AE}$ is formed by two components: an encoder $h(\cdot)$ that maps the input $x \in R_{d}$ to some hidden representation $h(x) \in R_{d} h$, and a decoder $g(\cdot)$, which maps the hidden representation back to a reconstructed version of the input $x$, so that $g(h(x)) \approx x$. Therefore, an AE is trained to minimize the discrepancy between the data and its reconstruction. This discrepancy represents the difference between the actual output vector and the expected output vector that is the same as the input vector. As a result, AEs offer a method to automatically learn features from unlabeled data, allowing for unsupervised learning.

One serious potential issue when working with $\mathrm{AE}$ is that if there is no other constraint besides minimizing the reconstruction error, then an AE with $n$ inputs and an encoding of dimension at least $n$ could potentially just learn the identity function, for which many encodings would be useless, leading to just copy the input. That means that an AE would not differentiate test examples from other input configurations. There are different ways that an AE with more hidden units than inputs could be prevented from learning the identity, and still capture some valuable information about the input in its hidden representation. 


\section{Deep architecture}

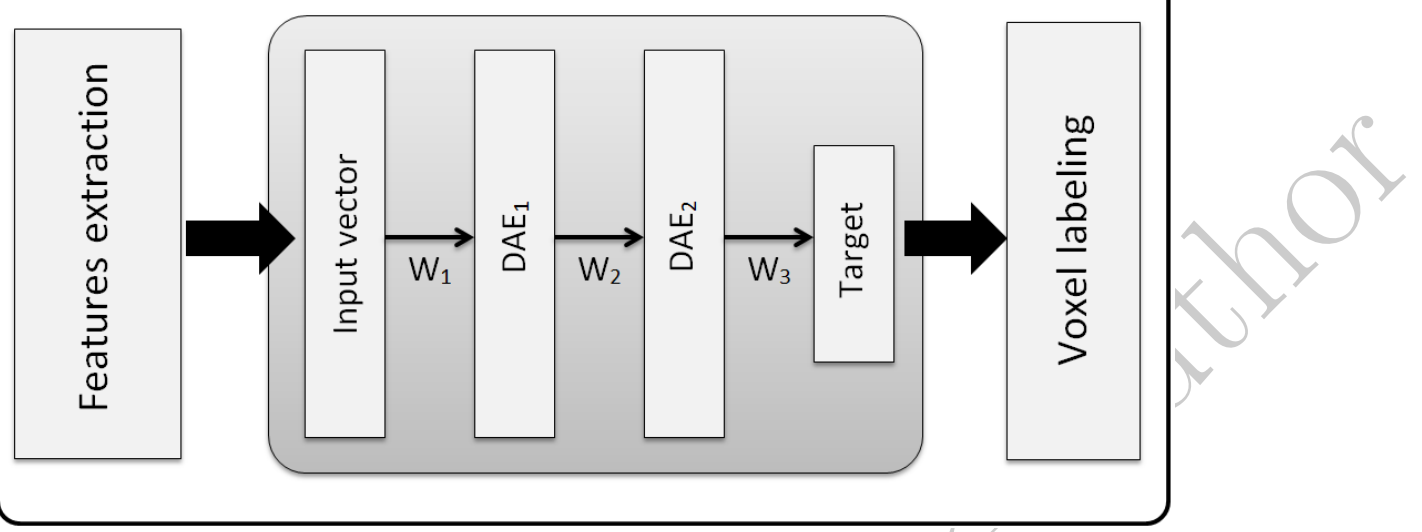

Figure 6: Deep network architecture constructred by stacking denoising autoencoders in the proposed approach.

Adding randomness in the transformation from input to reconstruction is one option, which is exploited in Denoising Auto-Encoders (DAEs) 16, 17.

The denoising auto-encoder (DAE) is typically implemented as a one-hiddenlayer neural network which is trained to reconstruct a data point $x \in \Re{ }^{D}$ from its corrupted version $\tilde{x}$ [17. This leads to a partially destroyed version $\tilde{x}$ by means of a stochastic mapping $\tilde{x} \sim q_{D}(\tilde{x} \mid x)$. Therefore, to convert an AE class into a DAE class, only adding a stochastic corruption step that modifies the input is required, which can be done in many ways. For example, in [17, the stochastic corruption process consists in randomly setting some of the inputs to zero. Several DAEs can be stacked to form a deep network by feeding the hidden representation of the DAE found on the layer below as input to the current layer [16. The unsupervised pre-training of such architecture is done greedily, i.e. one layer at a time. Each layer is trained as a DAE by minimizing the reconstruction of its input. Once the first $k$ layers are trained, the $(k+1)^{t h}$ layer can be trained because the latent representation from the layer below can be then computed.

In our network, DAEs are stacked to form the intermediate layers of the deep network (See Figure 6). More specifically, 2 hidden layers composed by 100 and 19 units, respectively, are used. During the unsupervised pre-training, the weights vectors $\left\{W_{1}, W_{2}\right\}$ are initially learned. Denoising corruption level

230 for the DAEs is set to 0.5 , since a value of $50 \%$ of noise level has already been proved to perform well in other problems [16.

\subsubsection{Supervised fine-tuning of the deep network}

Afterwards, when all layers have been pre-trained, the network goes through a second stage of training called fine-tuning, where prediction error is minimized on a supervised task. Weights vectors $\left\{W_{1}, W_{2}\right\}$ are already known from the 
previous step. The weights $\left\{W_{3}\right\}$ are now randomly initialized and convergence of the deep network is achieved via supervised learning, using the target class. During this process, weights $\left\{W_{1}, W_{2}\right\}$ are updated to tune the entire network. The hope is that the unsupervised initialization in a greedy layer-wise fashion has put the parameters of all the layers in a region of parameter space from which a good local optimum can be reached by local descent.

Following the same architecture than in the unsupervised pre-training, two hidden layers of DAEs are used, with the same number of units than before. At the end of the last layer of DAEs a softmax regression layer is used as output

245 with the sigmoid function as activation function. Mini-batch learning is followed during both unsupervised pre-training of DAEs and supervised fine-tuning of the entire network.

\subsection{Study design and experiment set-up}

\subsubsection{Dataset}

Image segmentation in the medical domain lacks from a universal known ground truth. Therefore, to validate segmentation approaches in clinical context, a number of observers and target patients that provide a good statistical analysis is required. Accordingly, this study has been designed to quantify variation among clinicians in delineating the brainstem and to assess our proposed classification scheme in this context. MRI data from 9 patients who underwent Leksell Gamma Knife Radiosurgery were used for training and leave one out cross validation. Pathologies in this dataset included trigeminal neuralgia, metastases, and brainstem cavernoma (See figure 7 for some visual examples). For each patient, the brainstem was manually delineated by four observers: 260 two neurosurgeons, one physician and one medical physicist. All of them were trained and qualified for radiosurgery delineation. Protocol for delineation was described before contouring session. The brainstem was delineated from cerebellar peduncle to occipital hole including the aqueduct of Sylvius. From manual segmentations of each patient a ground truth was generated by using majority voting rule with a threshold fixed at $75 \%$ of experts agreement. This ground truth was used to analyze deviations between the observers delineations, as well as the performance of the presented automatic approach. Properties of brainstem appearance across the 9 patients is shown in Table 1 . Artiview $® 3.0$ (Aquilab) was used after a training session to achieve Dicom RT contouring 270 structures. Average time of manual contouring was $20.2 \mathrm{~min}$ (SD: $10.8 \mathrm{~min}$ ). Two different MRI facilities were used to acquire images according to the radiosurgery planning protocol (Table 2). Particularly, MR T1 sequences were employed in this work.

In the proposed approach, and as in [9], before any process, MRI T1 images 275 were spatially aligned such that the anterior commissure and posterior commissure $(\mathrm{AC}-\mathrm{PC})$ line was horizontally oriented in the sagittal plane, and the inter hemispheric fissure was aligned on the two other axes. 

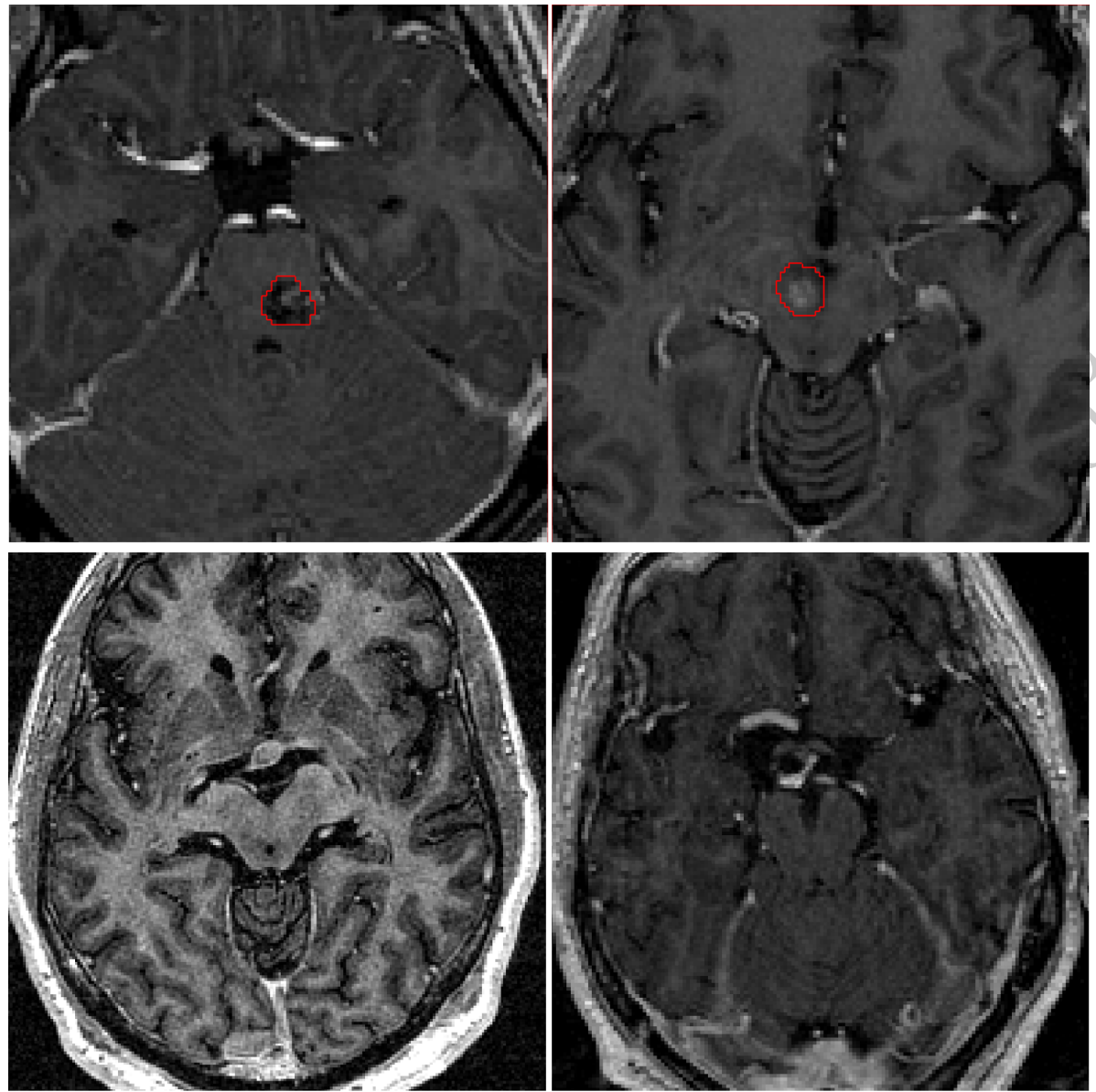

Figure 7: Some examples of images contained in the database employed in this work. While in some cases the tumor is inside the brainstem and may change the shape and intensity properties of the brainstem (top-row), in other brain cancer cases, tumors do not affect the brainstem properties.

\subsubsection{Evaluation}

Evaluation methods have lacked consensus as to comparison metrics. Since each metric yields different information, their choice is important and must be considered in the appropriate context. Although volume-based metrics, such as DSC [18, have been broadly used to compare volume similarities, they are fairly insensitive to edge differences when those differences have a small impact on the overall volume. Therefore, two segmentations with high degree of spa-

285 tial overlapping may exhibit clinically relevant differences at the edges. As a consequence, volume-based -i.e. DSC and percentage volume difference- and distance-based -i.e. Hausdorff distance- metrics are used to evaluate the segmentation results. Within-subjects analysis of variance (ANOVA), also referred 


\begin{tabular}{|c|c|c|c|c|c|}
\hline & \multicolumn{5}{|c|}{ Brainstem characteristics } \\
\hline & \multicolumn{4}{|c|}{ Intensity } & Volume \\
\hline & Mean & Standard deviation & Max & Min & cm3 \\
\hline Pat001 & $1,017.26$ & 170.36 & $3,426.00$ & 119.00 & 26.866 \\
\hline Pat002 & 221.91 & 28.23 & 326.00 & 32.00 & 22.894 \\
\hline Pat003 & 205.89 & 33.49 & 493.00 & 10.00 & 24.338 \\
\hline Pat004 & 168.05 & 29.72 & 456.00 & 14.00 & 27.241 \\
\hline Pat005 & 206.02 & 21.46 & 370.00 & 58.00 & 24.949 \\
\hline Pat006 & 173.14 & 19.12 & 326.00 & 33.00 & 21.114 \\
\hline Pat007 & 170.50 & 28.31 & 693.00 & 14.00 & 26.541 \\
\hline Pat008 & 209.77 & 23.15 & 351.00 & 35.00 & 27.437 \\
\hline Pat009 & 153.07 & 23.18 & 270.000 & 14.00 & 30.809 \\
\hline
\end{tabular}

Table 1: Properties of the brainstem across the patients of the dataset employed in this study.

\begin{tabular}{l|cccccc}
\hline MRI System & $\mathrm{TE}(\mathrm{ms})$ & $\mathrm{TR}(\mathrm{ms})$ & $\begin{array}{c}\text { Echo } \\
\text { number }\end{array}$ & Matrix size & Seq. Name & $\begin{array}{c}\text { Voxel Size } \\
\left(\mathrm{mm}^{3}\right)\end{array}$ \\
\hline $\begin{array}{l}\text { Philips Achieva } \\
\text { 1.5T }\end{array}$ & 4.602 & 25 & 1 & $256 \mathrm{x} 256$ & T1 3D FFE & $1 \mathrm{x} 1 \mathrm{x} 1$ \\
$\begin{array}{l}\text { GEHC Optima } \\
\text { MR450w 1.5T }\end{array}$ & 2.412 & 5.9 & 1 & $256 \times 256$ & FSPGR & $1 \times 1 \times 1.2$ \\
\hline
\end{tabular}

Table 2: Acquisition parameters on the 2 MRI devices.

to as repeated measures, on shape and volume similarity metrics followed by be used are extracted, and before training or testing, they are normalized to $[-1,1]$. These features vectors represent the input of both the SVM and deep learning schemes.

All the implementation was done in MATLAB. To perform the SVM classification the library libsvm [19] was used. To map the features into higherdimensionality spaces, a radial basis function (RBF) kernel was employed. To tune the other SVM parameters, as in the work of Dolz et al. [11, a grid search strategy was employed. Thus, values for SVM parameters $-C$ and $\gamma$ - were 7.5 310 and 3, respectively. Additionally, the implementation provided by Palm 20 . was used for the deep learning classification scheme. A workstation with $8 \mathrm{~GB}$ RAM and Intel Xeon processor at 3.06 was employed. 


\section{Results}

\subsection{Shape similarity}

The Dice similarity coefficient (DSC) was calculated for the four manual annotations and the three automatic methods. Mean DSC values for the four observers ranged from 0.84 to 0.90 , with minimum and maximum values of 0.78 and 0.93 respectively. Reference SVM $\left(\mathrm{SVM}_{1}\right)$ provided a mean DSC of 0.88 . On the other hand, whereas mean DSC for SVM with the proposed features was 0.91, the proposed deep learning based scheme reported a mean DSC of 0.92 (Fig. 8). The within-subjects ANOVA test conducted on the DSC of all the groups $(\mathrm{p}<0.05)$ indicated that there were significative differences among them. These differences were especially notorious on observers 1 and 4 .

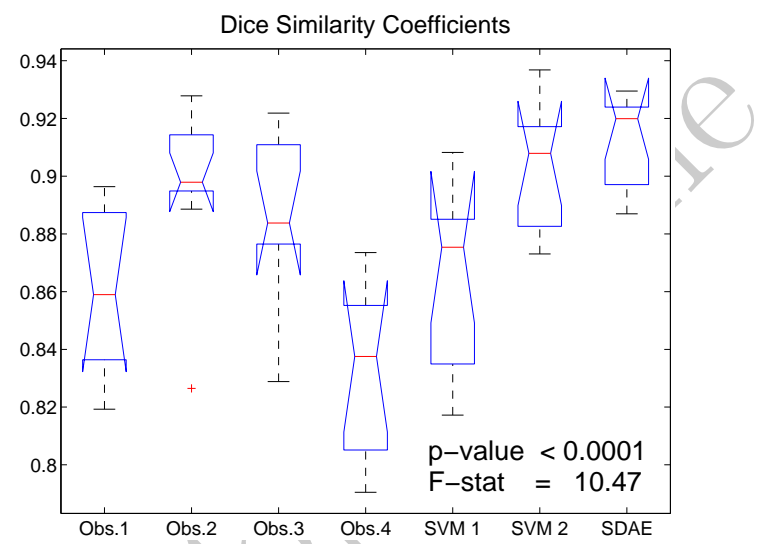

Figure 8: Segmentation DSC results for the observers and the automatic approaches.

Particularly for the machine and deep learning based approaches, a second set of ANOVAs was conducted to evaluate statistical differences between automatic methods. First, a within-subjects ANOVA (Figure 9, left) including the three methods indicated that there were significant differences between them. Therefore, at least one method significantly differed from the others $(\mathrm{p}<0.05)$. The post-hoc analysis (Bonferroni) confirmed the statistical differences between 330 these three approaches (see Figure 9, right). Paired repeated measures ANOVAs (Table 3) pointed out that, while approaches including the proposed features $\left(\mathrm{SVM}_{2}\right.$ and SDAE) performed significantly better $(\mathrm{p}<0.05)$ than $\mathrm{SVM}_{1}$, no significant differences were found between them $(\mathrm{p}=0.0811)$.

\begin{tabular}{|c|c|c|c|}
\hline & SVM $_{1}$ vs. $\mathrm{SVM}_{2}$ & $\mathrm{SVM}_{1}$ vs. SDAE & $\mathrm{SVM}_{2}$ vs. SDAE \\
\hline$p$-value & 0.0002 & 0.0001 & 0.0811 \\
\hline
\end{tabular}

Table 3: Within-subjects ANOVA on DSC values between automatic segmentation approaches.

Results also showed that Hausdorff distances decreased in the classification schemes using both machine and deep learning techniques, in comparison with manual segmentations (Fig. 10). Additionally, the addition of the proposed 

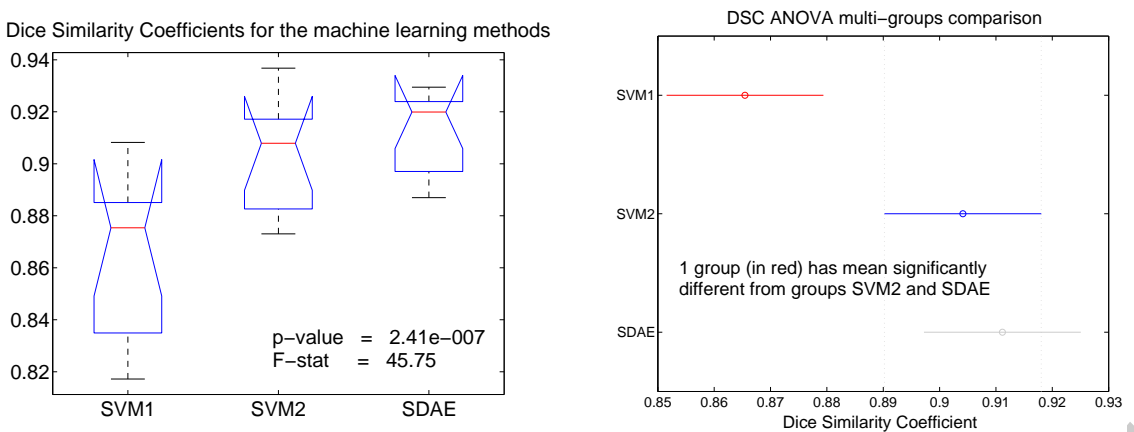

Figure 9: Within-subjects ANOVA analysis of the Dice score coefficients provided by the three automatic methods.

features $\left(\mathrm{SVM}_{2}\right.$ and $\left.\mathrm{SDAE}\right)$ decreased more the Hausdorff distances than the machine learning scheme used as reference, which did not include the suggested features $\left(\mathrm{SVM}_{1}\right)$. The within-subjects ANOVA test conducted on the Hausdorff distances values indicated that, although less significative than in the case of DSC, statistical differences between the groups existed. Again, these differences came overall from observer 1 and 4 . Taking into account the Hausdorff distances measured only on the machine and deep learning based schemes, a $p$-value of 0.0353 was obtained. As in the case of DSC values, at least one method significantly differed from the others $(\mathrm{p}<0.05)$. Paired repeated measures ANOVAs (Table 4 ) indicated that method $\mathrm{SVM}_{1}$ was statistically different from methods $\mathrm{SVM}_{2}$ and SDAE $(<0.05)$. Nevertheless, it failed to demonstrate a statistically significant difference between these two approaches $(\mathrm{p}=0.7874)$.

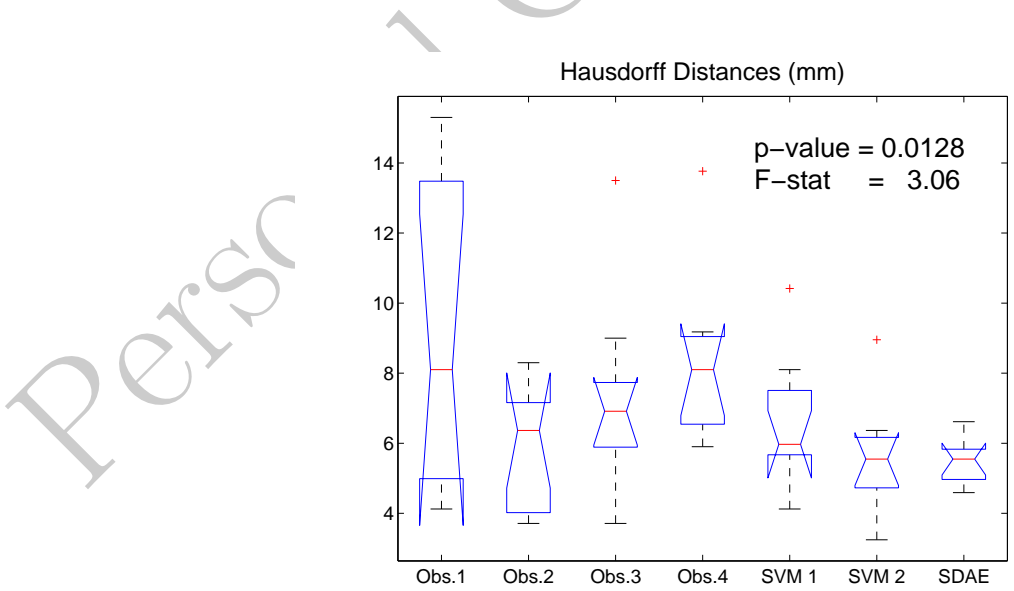

Figure 10: Hausdorff distances meassured for the volume segmented by the observers and the automatic approaches. 


\begin{tabular}{|c|c|c|c|}
\hline & SVM $_{1}$ vs. $\mathrm{SVM}_{2}$ & SVM $_{1}$ vs. SDAE & SVM $_{2}$ vs. SDAE \\
\hline$p$-value & 0.0067 & 0.0202 & 0.7874 \\
\hline
\end{tabular}

Table 4: Paired within-subjects ANOVA on Hausdorff distances between automatic segmentation approaches.

\subsection{Volume similarity}

Manual contours differed from the ground truth between 18.9\% (Observer 2) and $39.4 \%$ (Observer 4) as average (Table 5). In contrast, the three automatic approaches presented here largely decreased these volume differences, with a mean difference of $7.2 \%, 4.0 \%$ and $3.1 \%$ for $\mathrm{SVM}_{1}, \mathrm{SVM}_{2}$ and SDAE, respectively. Individual values for volume differences (\%) are shown in Table 5 for all the patients contoured by the four observers and the three analyzed methods. As it can be observed, classification schemes including the proposed features decreased the volume difference of automatic segmented volumes with respect to the reference ones.

\begin{tabular}{cccccccc}
\hline Patient & & \multicolumn{7}{c}{ Volume difference (\%) } & \multicolumn{1}{c}{ Y } \\
\hline & Obs.1 & Obs.2 & Obs.3 & Obs.4 & SVM $_{1}$ & SVM $_{2}$ & SDAE \\
$\# 1$ & $40.7 \%$ & $19.3 \%$ & $20.6 \%$ & $28.1 \%$ & $5.5 \%$ & $2.2 \%$ & $4.8 \%$ \\
$\# 2$ & $28.8 \%$ & $34.9 \%$ & $39.6 \%$ & $32.4 \%$ & $3.6 \%$ & $-1.9 \%$ & $1.8 \%$ \\
$\# 3$ & $24.1 \%$ & $13.7 \%$ & $29.3 \%$ & $49.4 \%$ & $3.8 \%$ & $2.0 \%$ & $0.8 \%$ \\
$\# 4$ & $34.4 \%$ & $19.8 \%$ & $17.3 \%$ & $41.6 \%$ & $-4.4 \%$ & $5.2 \%$ & $3.1 \%$ \\
$\# 5$ & $18.7 \%$ & $13.9 \%$ & $15.5 \%$ & $51.7 \%$ & $-8.2 \%$ & $-6.6 \%$ & $-3.4 \%$ \\
$\# 6$ & $22.9 \%$ & $19.6 \%$ & $13.7 \%$ & $32.6 \%$ & $-7.6 \%$ & $-3.5 \%$ & $-3.7 \%$ \\
$\# 7$ & $33.3 \%$ & $15.8 \%$ & $25.3 \%$ & $33.5 \%$ & $-2.5 \%$ & $-3.6 \%$ & $-3.1 \%$ \\
$\# 8$ & $21.2 \%$ & $15.9 \%$ & $25.5 \%$ & $47.6 \%$ & $-12.6 \%$ & $-5.5 \%$ & $4.0 \%$ \\
$\# 9$ & $40.6 \%$ & $17.3 \%$ & $25.9 \%$ & $38.1 \%$ & $-16.2 \%$ & $-5.0 \%$ & $-3.2 \%$ \\
\hline Average & $29.4 \%$ & $18.9 \%$ & $23.6 \%$ & $39.4 \%$ & $7.2 \%$ & $4.0 \%$ & $3.1 \%$ \\
\hline Std. Dev & $8.3 \%$ & $6.4 \%$ & $8.0 \%$ & $8.5 \%$ & $4.6 \%$ & $1.7 \%$ & $1.2 \%$ \\
\hline
\end{tabular}

Table 5: Evaluation results (Volume difference (\%)) of the manual contouring for the four observers, SVM and proposed approach with respect to the generated ground truth. The average represents the average of the absolute of volume difference values.

A within-subjects ANOVA was performed over the volume differences values for the four observers and the three automatic contours (Figure 11). Visual difference between the groups of manual and automatic segmentations is confirmed by the $p$-value $(<0.05)$ obtained in the ANOVA test. Because the $p$-value was less than the significance level of 0.05 , the null hypothesis can be rejected and we can conclude that some of the groups have statistically significant differences 365 on their mean values. The post-hoc analysis (Bonferroni) is shown Figure 11 on the right. It confirms that significant differences came from the groups formed by the 4 observers in one side, and by the automatic contours in the other side.

Repeated measures ANOVA tests between volume differences and the ground truth was conducted to individually compare the volumes. Computed $p$-values 370 for the automatic segmentation methods were greater than 0.05 , particularly in the proposed deep learning scheme (Table 6). Thus, statistical results showed a 

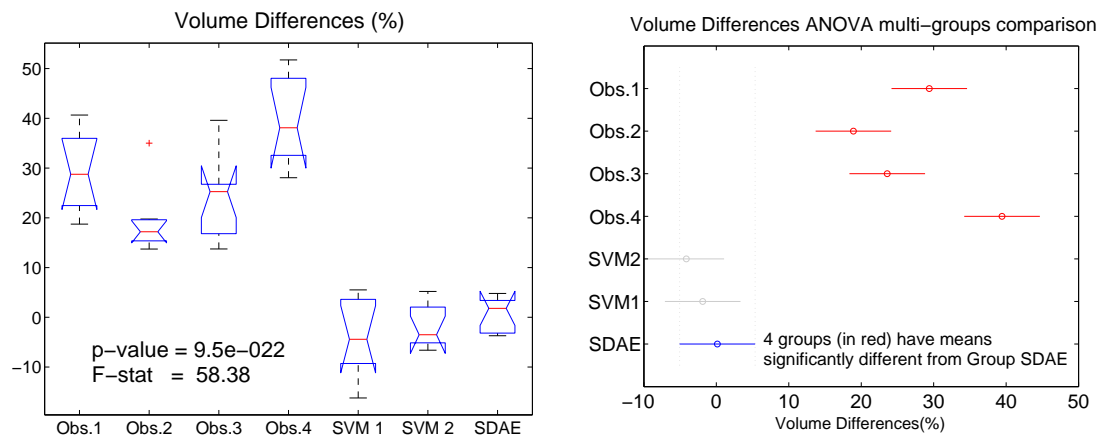

Figure 11: Results of the within-subjects ANOVA test conducted on the volume difference values for the four manual contours and the three automatic methods. On the left, mean volume differences and deviations are shown. On the right, the ANOVA multi-group comparison is displayed, where the automatic method SDAE is selected as reference of the comparison.

significant similarity between automatically segmented volumes and generated ground truth volumes, notably for the SDAE based scheme.

\begin{tabular}{|c|c|c|c|}
\hline & SVM $_{1}$ vs. GroundTruth & SVM $_{2}$ vs. GroundTruth & SDAE vs. GroundTruth \\
\hline$p$-value & 0.1175 & 0.1841 & 0.4584 \\
\hline
\end{tabular}

Table 6: Repeated measures ANOVA on volume differences values between automatic segmentation approaches and the ground truth.

Segmentation result on a given patient can be visually seen in Fig. 12 . On 375 this figure, both manual and automatic contours are displayed, in addition to the ground truth. It can be observed that contours generated by the automatic approach lie on the variability of the four experts.

\subsection{Classification time}

Regarding the segmentation time of the automatic approaches, while the features extraction time was the same for all the approaches, classification time differed between the SVM and SDAE based schemes. Features extraction process was done in around 15 seconds as average for each volume. Concerning the segmentation, layouts based on SVM reported a mean classification time close to 25 seconds for the whole volume. Contrary, the deep learning-based scheme 385 performed the task in 0.36 seconds as average.

\section{Discussion}

A deep learning-based classification scheme formed by stacking denoising auto-encoders has been proposed in this work to segment the brainstem. It has been compared with a machine learning approach widely and successfully used 
distance transform map, and a modified version of a 3D local binary pattern has been proposed and evaluated.

The most influencing factor in realizing a classifier with high generalization in the classifier may lead to unsatisfactory results. Ideal features should bring a high discriminative power to the classifier. Features proposed in this work have different nature. While some of them come from pure image intensity properties, some others provide spatial or texture knowledge. Thus, they are 'a these features, classification tests were conducted by using only pairs of features (See fig. 4). Although it was not presented, combination of all of these features together improves the classification task, creating a classifier with higher discriminative power. This improvement on classification is reflected in the results section

Statistical analysis indicated that there were significant differences between the automatic (machine and deep learning based) schemes and the manual delineations made by the four experts. In addition, the ANOVA tests performed between the machine and deep learning based approaches, suggested that differences between them were statistically significatives on the DSC evaluation. These differences were particularly important in the classification schemes that included the proposed features. Although differences were less significative in the rest of the similarity metrics, results showed that our deep learning classification scheme performed better than the other machine learning based ap415 proaches. In terms of segmentation time, while features extraction was equal in all the approaches, classification time reported by the deep learning scheme was approximately 70 times faster than SVM based scheme. Consequently, the proposed deep learning architecture demonstrated a significant gain in the performance of the brainstem segmentation on MRI, outperforming the widely used SVM approach.

Even though the presented work is not pioneering on the evaluation of automatic segmentation of the brainstem, among others, in the context of radiation therapy, it presents important improvements respect to the others (See Table 7). All these previous methods are atlas-based and thus registration dependent. ${ }_{425}$ This makes segmentation times to be over several minutes, which might be clinically impractical in some situations. Our method, however, performs the segmentation in few seconds. A noteworthy point is that features extraction represented nearly $97.5 \%$ of the whole segmentation process. Since this stage is composed by simple and independent image processing steps, this can be eas430 ily parallelized. By doing this, the total segmentation time may be drastically reduced.

Results provided in this work demonstrated that the proposed deep learningbased classification scheme outperformed all previous works when segmenting

\footnotetext{
${ }^{1}$ These two approaches required registration steps which took 20 minutes in the first case and around 3 minutes for the second method.
} 


\begin{tabular}{|c|c|c|c|c|}
\hline Reference & Method & DSC & pVD (\%) & Segmentation Time \\
\hline Bondiau et al.,2005 & Atlas-Based & - & -13.11 & $20 \mathrm{~min}$. \\
\hline Isambert et al.,2008 & Atlas-Based & 0.85 & -14.8 & 7-8 min. (6 OARs) \\
\hline Babalola et al.,2009 & $\begin{array}{c}\text { Atlas-Based } \\
\text { Statistical-Based (PAM) } \\
\text { Statistical-Based (BAM) } \\
\text { Expectation-Minilization }\end{array}$ & $\begin{array}{l}0.94 \\
0.88 \\
0.89 \\
0.83\end{array}$ & $\begin{array}{c}3.98 \\
6.80 \\
7.80 \\
21.10\end{array}$ & $\begin{array}{c}\text { 120-180 min. (Set of brain structures) } \\
1 \text { min. }+20 \text { min. }{ }^{1} \\
5 \text { min. }+3 \text { min } 1 \\
30 \mathrm{~min} \text {. (Set of brain structures) }\end{array}$ \\
\hline Deeley et al.,2011 & Atlas-Based & 0.85 & - & - \\
\hline Dolz et al.,2015 & Support Vector Machines & 0.88 & 7.2 & $(15+25)$ seconds \\
\hline Proposed scheme & Stacked Denoising Auto-encoders & 0.91 & 3.08 & $(15+0.36)$ seconds \\
\hline
\end{tabular}

Table 7: Table that summarizes results of previous works which attempted to segment the brainstem on MRI images. DSC and pVD are given as mean values.

the brainstem. Furthermore, the addition of the novel features, i.e. geodesic

distance transform map and LBP-3D, in the classifier increased the volume similarity at the same time that reduced Hausdorff distances. Nevertheless, it is important to note that differences in data acquisition, as well as metrics used to evaluate the segmentation, often compromise comparison to other works. More important than the improvements with respect to other methods, is the

440 clinical validation in regard to variability between clinically adopted contours. When comparing the results with the manual contours, it can be observed that they lie inside the variability of the observers. This fact, together with the remarkably low segmentation time reported, makes this technique suitable for being used in clinical routine. Therefore, the introduction of such technique may help radiation oncologists to save time during the RTP, as well as reducing variability in OAR delineation.

One of the strengths of machine and deep learning methods relies on their ability to transfer knowledge from human to machine. Hence, for example, when no visible boundaries are present, the classifier uses its transferred intelligence 450 from doctors to perform the segmentation as they would do. As example, we can cite the area where the brainstem meets the cerebellum in the lower pons (See Fig. 1, left). No contrasted and visible boundary is present in this region, so experts use their knowledge and experience to delineate the brainstem contours. Clinically speaking, the contour starts anteriorly at the basilar sulcus of the ponds and it is extended laterally to include the middle cerebellar peduncles. The contour continues then posteriorly and medially towards the median sulcus of the fourth ventricle. This would not be possible without the experts' knowledge. Therefore, transferring their acquired knowledge to the deep architecture to be learned helps in assisting to the delineation task in areas where 460 other methods would fail.

Extension of the proposed deep architecture approach to other organs at risk involved in the RTP, such as the optic chiasm or the cochlea, is envisaged. In addition to the information coming from MR-T1, intensity properties of MR-T2 may help in the segmentation process. Therefore, the impact of including inten465 sity values of MR-T2 images in the features vector will be investigated. Future work will also aim at validating our deep learning based scheme on a larger dataset with the ultimate goal of gradually bringing automated segmentation 
tools into clinical practice.

\section{Conclusion}

470 segment the brainstem on brain cancer patients. Particularly, we address this problem by stacking denoising auto-encoders in a deep network to learn the features representation of the network input. In addition to classical features employed in machine learning-based methods to segment brain structures, two new with the set of proposed features, our method showed a better performance than a state-of-the-art classification technique, i.e. SVM. Furthermore, results demonstrate that our method also achieved better results than other existing works. We also evaluated the proposed learning scheme in a clinical setting, method lie inside the variability of observers. Additionally, segmentation times reported for the automatic segmentations were remarkably low. All this makes our proposed approach suitable to be employed in clinical routine.

Acknowledgments. This project has received funding from the European 's Seventh Framework Programme for research, technological development and demonstration under grant agreement no PITN-GA-2011-290148.

\section{References}

1. J. Ferlay, H. Shin, F. Bray, D. Forman, C. Mathers, D. Parkin, Globocan 2008, cancer incidence and mortality worldwide: Iarc cancerbase no. 10, Lyon, France: International Agency for Research on Cancer 2010 (2010) 29.

2. M. Deeley, A. Chen, R. Datteri, J. Noble, A. Cmelak, E. Donnelly, A. Malcolm, L. Moretti, J. Jaboin, K. Niermann, et al., Comparison of manual and automatic segmentation methods for brain structures in the presence of space-occupying lesions: a multi-expert study, Physics in medicine and biology 56 (14) (2011) 4557.

3. P.-Y. Bondiau, G. Malandain, S. Chanalet, P.-Y. Marcy, J.-L. Habrand, F. Fauchon, P. Paquis, A. Courdi, O. Commowick, I. Rutten, et al., Atlasbased automatic segmentation of $\mathrm{mr}$ images: validation study on the brainstem in radiotherapy context, International Journal of Radiation Oncology* Biology* Physics 61 (1) (2005) 289-298.

4. K. O. Babalola, B. Patenaude, P. Aljabar, J. Schnabel, D. Kennedy, W. Crum, S. Smith, T. Cootes, M. Jenkinson, D. Rueckert, An evaluation of four automatic methods of segmenting the subcortical structures in the brain, Neuroimage 47 (4) (2009) 1435-1447.

505 5. J. Dolz, L. Massoptier, M. Vermandel, Segmentation algorithms of subcortical brain structures on mri for radiotherapy and radiosurgery: A survey (2015). doi:10.1016/j.irbm.2015.06.001 
6. A. Isambert, F. Dhermain, F. Bidault, O. Commowick, P.-Y. Bondiau, G. Malandain, D. Lefkopoulos, Evaluation of an atlas-based automatic segmentation software for the delineation of brain organs at risk in a radiation therapy clinical context, Radiotherapy and oncology 87 (1) (2008) 93-99.

7. Y. Guo, G. Wu, L. A. Commander, S. Szary, V. Jewells, W. Lin, D. Shen, Segmenting hippocampus from infant brains by sparse patch matching with deep-learned features, in: Medical Image Computing and ComputerAssisted Intervention-MICCAI 2014, Springer, 2014, pp. 308-315.

8. W. Zhang, R. Li, H. Deng, L. Wang, W. Lin, S. Ji, D. Shen, Deep convolutional neural networks for multi-modality isointense infant brain image segmentation, NeuroImage 108 (2015) 214-224.

9. S. Powell, V. A. Magnotta, H. Johnson, V. K. Jammalamadaka, R. Pierson, N. C. Andreasen, Registration and machine learning-based automated segmentation of subcortical and cerebellar brain structures, Neuroimage 39 (1) (2008) 238-247.

10. E. Y. Kim, H. Johnson, Multi-structure segmentation of multi-modal brain images using artificial neural networks, in: SPIE Medical Imaging, International Society for Optics and Photonics, 2010, pp. 76234B-76234B.

11. J. Dolz, A. Laprie, S. Ken, H.-A. Leroy, N. Reyns, L. Massoptier, M. Vermandel, Supervised machine learning-based classification scheme to segment the brainstem on mri in multicenter brain tumor treatment context, International Journal of Computer Assisted Radiology and Surgery (2015) 1-9doi:10.1007/s11548-015-1266-2

12. A. Criminisi, T. Sharp, A. Blake, Geos: Geodesic image segmentation, in: Computer Vision-ECCV 2008, Springer, 2008, pp. 99-112.

13. C. Montagne, A. Kodewitz, V. Vigneron, V. Giraud, S. Lelandais, et al., 3d local binary pattern for pet image classification by svm, application to early ${ }_{535}$ alzheimer disease diagnosis, in: Proc. of the 6th International Conference on Bio-Inspired Systems and Signal Processing (BIOSIGNALS 2013), 2013, pp. $145-150$.

14. C. J. Burges, A tutorial on support vector machines for pattern recognition, Data mining and knowledge discovery 2 (2) (1998) 121-167.

540 15. Y. Bengio, Learning deep architectures for ai, Foundations and trends $\AA$ in Machine Learning 2 (1) (2009) 1-127.

16. P. Vincent, H. Larochelle, I. Lajoie, Y. Bengio, P.-A. Manzagol, Stacked denoising autoencoders: Learning useful representations in a deep network with a local denoising criterion, The Journal of Machine Learning Research $545 \quad 11(2010) 3371-3408$. 
17. P. Vincent, H. Larochelle, Y. Bengio, P.-A. Manzagol, Extracting and composing robust features with denoising autoencoders, in: Proceedings of the 25th international conference on Machine learning, ACM, 2008, pp. 10961103.

550 18. L. R. Dice, Measures of the amount of ecologic association between species, Ecology 26 (3) (1945) 297-302.

19. C.-C. Chang, C.-J. Lin, LIBSVM: A library for support vector machines, ACM Transactions on Intelligent Systems and Technology 2 (2011) 27:1 $27: 27$.

20. R. B. Palm, Prediction as a candidate for learning deep hierarchical models of data, Technical University of Denmark, Palm 25. 

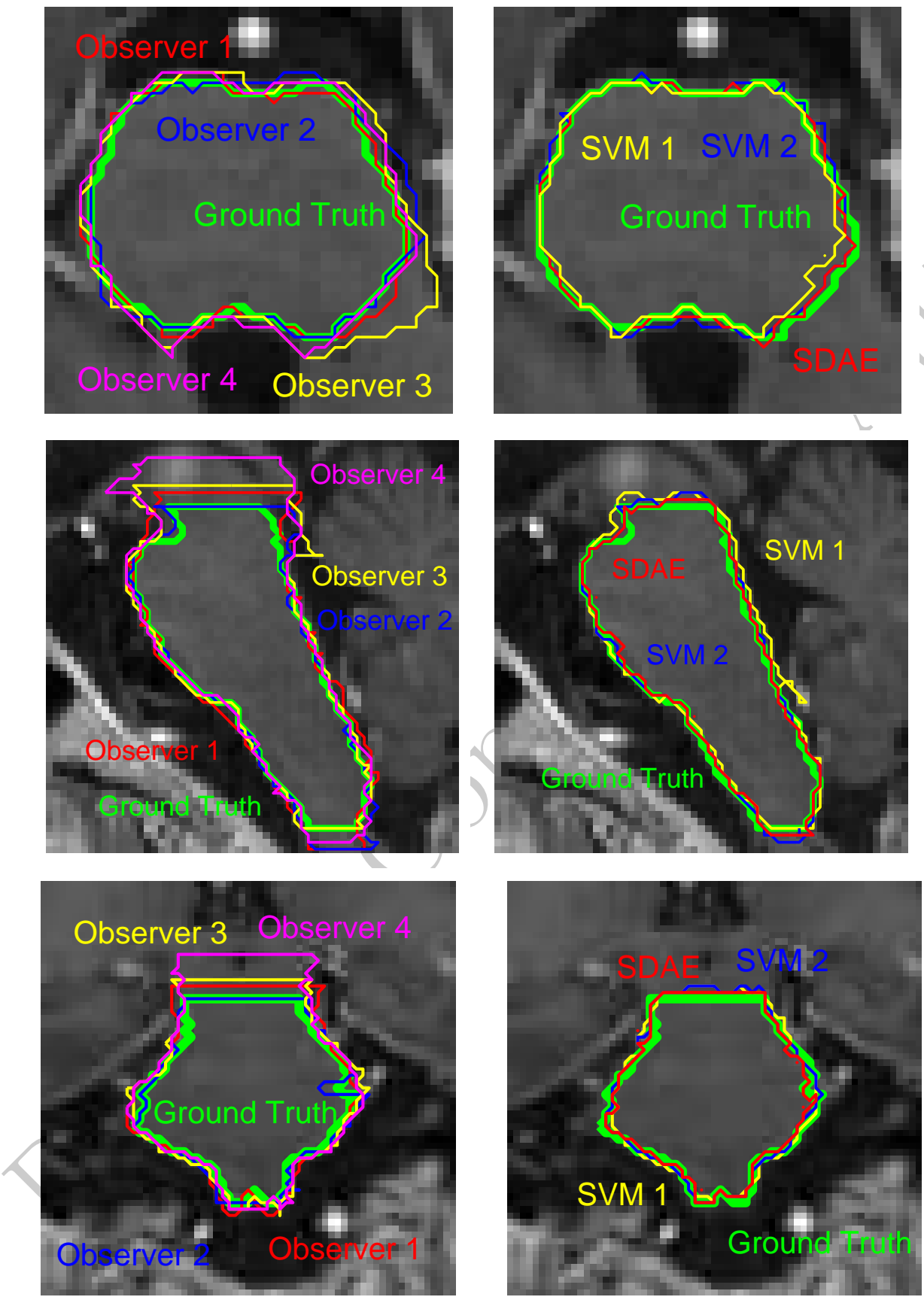

Figure 12: Contours for a given patient. Columns represent manual (first) and automatic (second) contours, while rows display axial, sagittal and coronal planes, respectively. In green is represented the generated ground truth. Manual and automatic contours are displayed in different colors, which are indicated in the images. 